\title{
Bilateral stenting of symptomatic and asymptomatic internal carotid artery stenosis due to fibromuscular dysplasia
}

\author{
J Finsterer, J Strassegger, A Haymerle, G Hagmüller
}

\begin{abstract}
Stent grafting of internal carotid artery (ICA) stenoses due to fibromuscular dysplasia has been rarely and only unilaterally carried so far. Bilateral carotid stent grafting of ICA stenoses due to fibromuscular dysplasia has not been reported previously. In a 37 year old woman with recurrent right hemispheric transitory ischaemic attacks, a non-disabling minor stroke, and recurrent right amaurosis fugax despite antithrombotic therapy, cerebral angiography disclosed a long segment narrowing, distal, high grade $(95 \%)$ stenosis of the right ICA and a long narrowing, distal high grade $(70 \%)$ stenosis of the left ICA. Morphological features of both stenoses were indicative of fibromuscular dysplasia. The right sided stenosis was stented with a PTFEHEMOBAHN ${ }^{\circledR}$ endoprosthesis; this was followed by a brief, postprocedural left sided hemiparesis. The left sided ICA stenosis was successfully stented by the same procedure. Nine months later, both stents were still patent and the patient was symptom free. Bilateral carotid stenting may remain an alternative to endarterectomy in bilateral ICA stenosis due to fibromuscular dysplasia when ischaemic events persist despite full antithrombotic therapy.

(F Neurol Neurosurg Psychiatry 2000;69:683-686)
\end{abstract}

Neurological

Department,

Neurological Hospital

Rosenhügel, Vienna,

Austria

J Finsterer

A Haymerle

Department of Vascular Surgery, Wilhelminenspital, Vienna, Austria

J Strassegger

G Hagmüller

Correspondence to: Dr J Finsterer, Sch 348, 1180 Wien, Austria fij@2nr.nkr.magwien.gr.at

Received 4 October 1999 and in revised form

19 April 2000

Accepted 21 June 2000
Keywords: carotid surgery; carotid dysplasia; internal carotid artery stenosis; angioplasty

Usually, prevention of stroke in patients with fibromuscular dysplasia of the internal carotid artery (ICA) is carried out with antiplatelet and anticoagulation therapy to prevent thrombus formation or by revascularisation of stenosed segments by carotid endarterectomy. ${ }^{1}$ Percutaneous endovascular techniques, in particular elective (primary) carotid stent grafting are becoming increasingly important as an alternative to carotid endarterectomy. ${ }^{1}$ So far, carotid stent grafting has been applied to dysplastic ICA stenosis only unilaterally on the symptomatic side. ${ }^{2-4}$ Bilateral carotid stent grafting of significant ICA stenoses due to fibromuscular dysplasia has not been reported before.
Case history

A 37 year old non-smoking, non-diabetic woman, with a long history of migraine, and occasionally high blood pressure not necessitating antihypertensive medication, and no history of anginal pain, developed hypaesthesia of the left arm for a few minutes, immediately after an abortion in October 1998. A similar episode of transient hypaesthesia occurred in December 1998. A few hours later, a left central 7th cranial nerve palsy with concomitant amaurosis fugax of the right eye occurred for a few minutes. She reported that she had had several episodes of right sided amaurosis fugax for 4 years. She was referred for suspected multiple sclerosis, but on clinical neurological examination there was an exclusively left sided accentuation of deep tendon reflexes. Blood chemistry showed slight anaemia. Neck ultrasonography examination of the extracranial cerebral vessels disclosed a high grade $(95 \%)$ ICA stenosis on the right side and a further high grade $(70 \%)$ ICA stenosis on the left side. Cerebral angiography located the right sided stenosis $2 \mathrm{~cm}$ before entry into the skull base and the left sided stenosis distally to the angulus mandibulae (fig 1). Proximally to both stenoses the arterial wall was irregularly shaped, including narrowings and widenings over a long segment (fig 1). Although dissection, thrombus formation, and dissecting aneurysm were considered as an explanation for the angiographic findings, the "string of beads"-like impression was interpreted as fibromuscular dysplasia in the last analysis. There was no indication for fibromuscular dysplasia in the iliac or renal arteries. T1 weighted MRI of the brain showed a hyperintense white matter lesion involving only a small part of the right medial cerebral artery territory, laterally to the right lateral ventricle. Transthoracic echocardiography was normal. Acetylsalicylic acid (100 mg/day) was started. In January 1999 the patient underwent endovascular surgery of the right sided stenosis of the middle segment of the extracranial ICA. A $5 \mathrm{~cm} \times 6 \mathrm{~mm}$ PTFEHEMOBAHN ${ }^{\circledR}$ endoprosthesis (WL Gore and Ass, Flagstaff, AZ, USA) was inserted via transverse neck skin incision and open puncture of the common carotid artery (CCA, fig 2). The operation was performed under locoregional anaesthesia supported by intraop- 

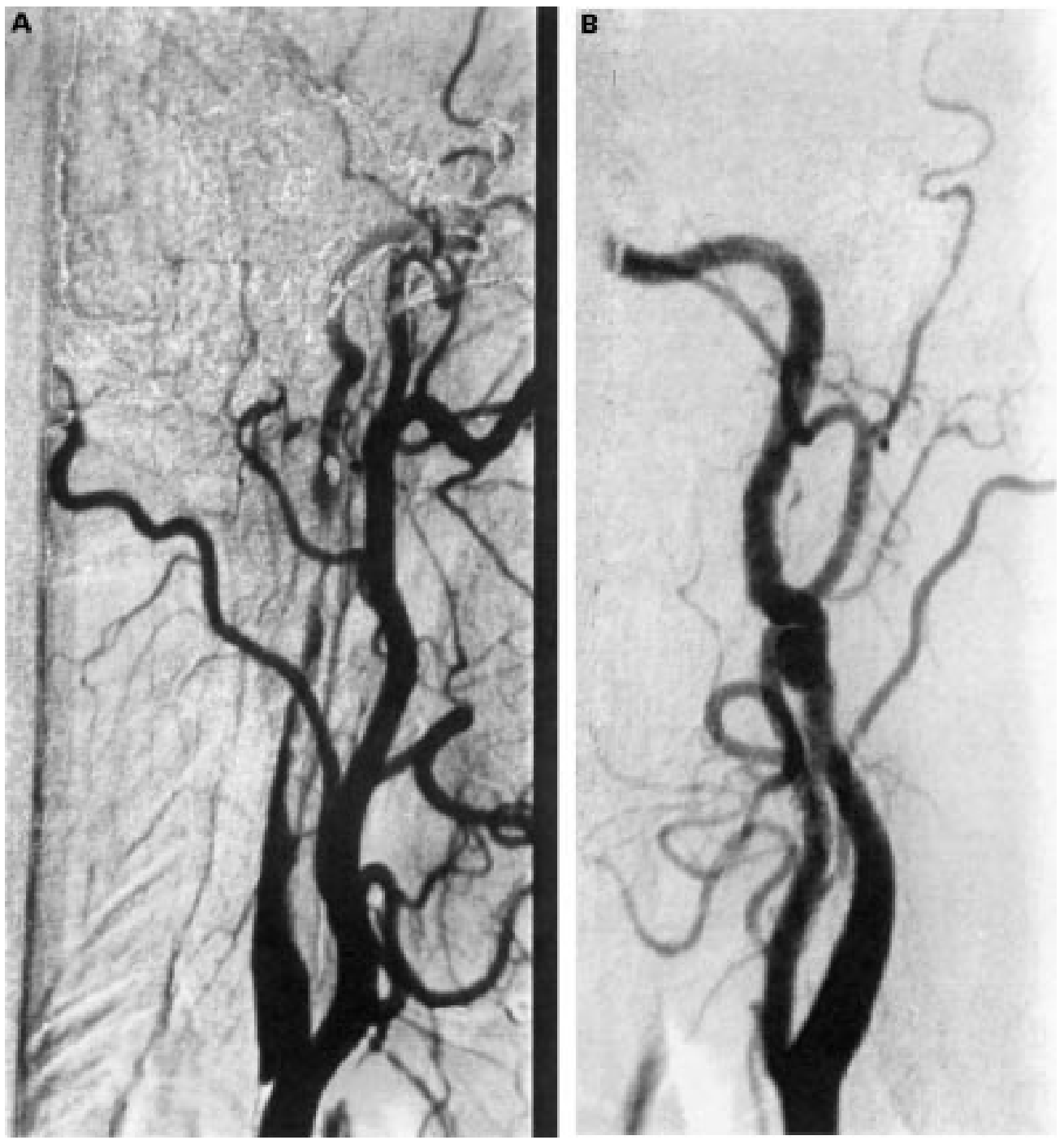

Figure 1 Cerebral angiography shows long segment narrowing, high grade (95\%) stenosis of the right ICA about $2 \mathrm{~cm}$ before entry into the skull base (left panel) and a $70 \%$ stenosis of the left ICA (as confirmed by ultrasound), distally to the angulus mandibulae (right panel). The arterial walls are irregularly shaped, including widening and narrowing of the arterial lumen.

erative digital subtraction angiography fluoroscopy. The blood flow was interrupted by central clamping of the CCA to prevent intraoperative cerebral embolism during balloon dilation and endograft insertion in this long segmented ICA stenosis. Clamping time was 9 minutes. Before declamping the CCA, the 8-F introducer system, through which the endovascular manipulation in the ICA was performed, was retrogradely flushed with blood to prevent cerebral embolisation of possible intravascular debris from the manipulated area of the ICA. Despite these measures, a slight left sided hemiparesis occurred for 1 hour immediately after the procedure. This postoperative hemiparesis on the symptomatic side was explained with the application of a non-standard procedure and the relatively long clamping time. No further postprocedural deficit was noted. Forty seven days later, the asymptomatic left sided ICA stenosis was stented in the same manner (fig 2). The postprocedural course after the second intervention was uneventful. Three, 6 , and 9 months after the second procedure, ultrasonography confirmed contiguous patency of both stents and the patient was symptom free.

\section{Discussion}

Fibromuscular dysplasia is a non-atherosclerotic non-inflammatory arteropathy that affects the medium sized arteries (carotid, iliac, renal). Its prevalence is $0.5-0.7 \%$ by angiography. ${ }^{56}$ The characteristic angiographic features of fibromuscular dysplasia are alteration of expanded and narrowed zones of the arterial lumen ("string of beads") and carotid lengthening that entails tortuosity, coiling, and kinking. ${ }^{7}$ Usually, the distal part of the ICA is involved, whereas the proximal part is spared. ${ }^{5}$ In $85 \%$ of the cases fibromuscular dysplasia is bilateral, and $85 \%$ of the cases are middle aged women. ${ }^{8}$ fibromuscular dysplasia occurs in conjunction with various connective tissue disorders, and is assumed to be a non-specific entity. ${ }^{9}$ The pathogenic background of fibromuscular dysplasia is so far unknown, but there are indications that it is due to vasa vasorum 

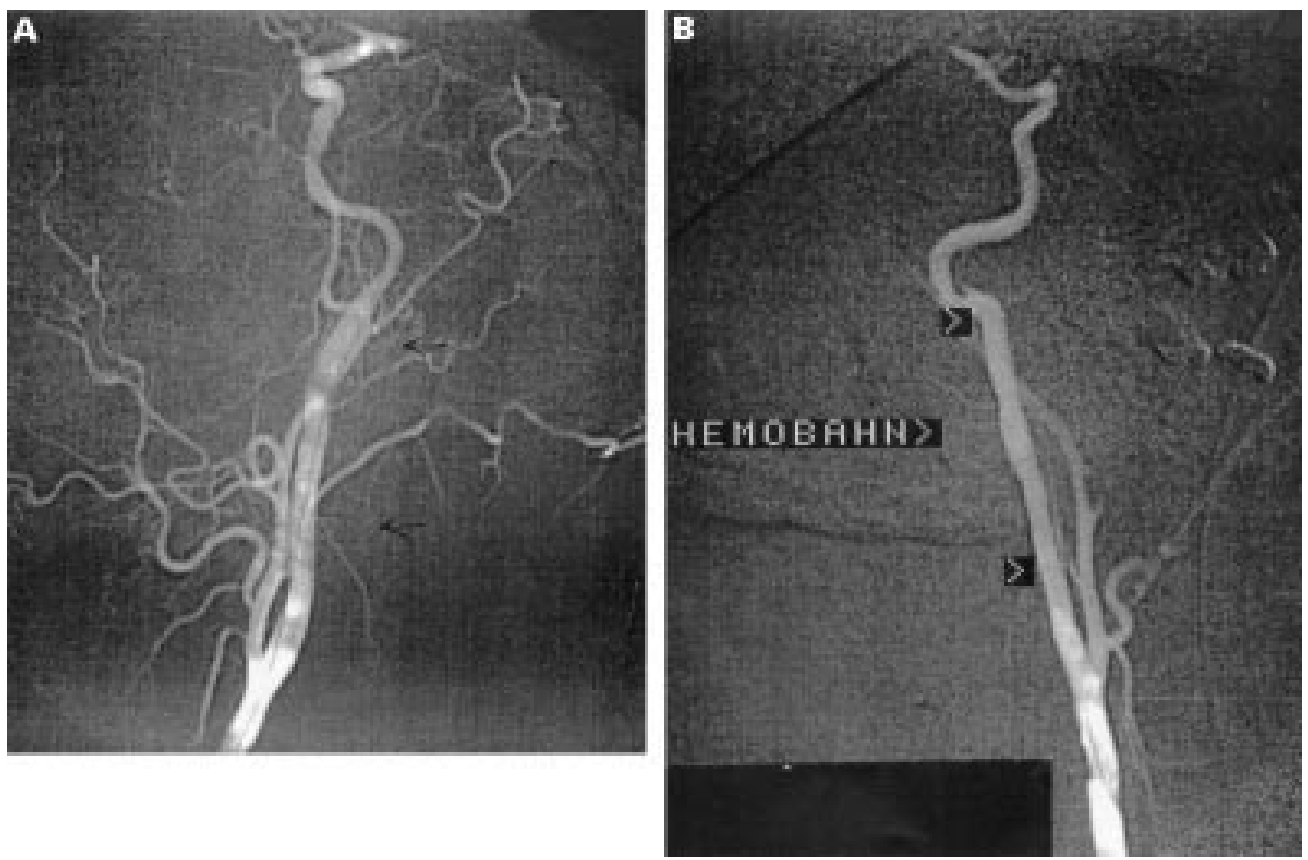

Figure 2 Final intraoperative angiography of the right (left panel) and left (right panel) ICA after successful stent grafting shows continuous patency of both endografts.

paucity, repeated microtrauma, inadequate hormonal background, or $\alpha$-antitrypsin deficiency. ${ }^{7}$ It is implicated in the cause of transitory ischaemic attacks, stroke, and subarachnoidal bleeding. ${ }^{10}$

For years the standard therapy to prevent strokes in patients with fibromuscular dysplasia of the ICA was the application of antithrombotic therapy or carotid endarterectomy. Since the 1980s, carotid stent grafting has been proposed as an alternative to carotid endarterectomy and is growing in importance. ${ }^{11}{ }^{12}$ Principally, stenting can be carried out with self expanding stents (for example, Wall stents) or stents deployed after prior balloon dilatation of the stenosed segment (for example, PalmazSchatz stents). ${ }^{1}$ Stenting has a high technical success rate, of up to $99 \%,{ }^{12-15}$ has periprocedural complication rates of $<6 \%$, similar to carotid endarterectomy, ${ }^{16}$ and a 6 month restenosis rate of $<5 \% .{ }^{17}{ }^{18}$ Although still under debate and intensive investigation, carotid stent grafting is indicated in (1) the correction of significant ICA stenosis not readily accessible to surgery, (2) recurrent ICA stenosis after carotid endarterectomy, (3) non-atherosclerotic lesions, (4) patients with high surgical morbidity and mortality (age $>85$ years, coronary heart disease, malignoma, contralateral occlusion) or polymorbidity, (5) multiple simultaneous endovascular interventions, and (6) patients with a "hostile neck" with scars after radiation, trauma, or burns. ${ }^{1}$ Although carotid stent grafting seems to be technically feasible, safe, and provides a satisfactory short term patency, little is known about the durability of the benefit and the long term restenosis rate; hence randomised trials have to be carried out before carotid stent grafting can be offered as an alternative to carotid endarterectomy. ${ }^{13}$ 17-19
Carotid stent grafting was chosen as the treatment of choice for the symptomatic ICA stenosis in our patient because of the nonatherosclerotic nature, multiple segment extension, a periprocedural complication rate similar to carotid endarterectomy, the presumably underlying dissection with a high probability of developing aneurysms, its low invasiveness, and the recent promising reports about successful stenting of ICA stenoses due to fibromuscular dysplasia. ${ }^{2-4}$ DeOcampo et al reported on one patient with occlusive dissection of the left dysplastic ICA in whom the vessel was opened by carotid stent grafting, with marked clinical improvement. ${ }^{2}$ Manninen et al reported on a patient with haemorrhage, cervicocranial fibromuscular dysplasia, and unilateral ICA dissection, who was successfully given multiple stents. ${ }^{3}$ Starr et al reported on 25 patients with fibromuscular dysplasia of whom one was treated with carotid stent grafting but had a non-disabling stroke during stent placement. ${ }^{4}$ Although carotid stent grafting of non-symptomatic ICA stenosis is a nonstandard practice, it was also applied to the asymptomatic left ICA stenosis, because of its high grade, the high probability of impending dissection, development of aneurysms, thrombus formation, occlusion, its nonatherosclerotic nature, its distal location, its extension, and the successful carotid stent grafting on the right side.

Carotid stent grafting was chosen as the intervention of choice on both sides because it is not yet clear if carotid endarterectomy and carotid stent grafting have similar long term success rates. ${ }^{19}$ The neck cut down access was chosen because the common carotid artery is clamped proximal to the puncture site, thus reducing the probability of embolisation from a pulsating artery, and debris, produced during 
the procedure, are continuously removed by suction. The PTFE endoprosthesis was chosen because (1) the open netting wire of the Palmaz and Wall stents promote microembolisation, (2) the inner layer of the PTFE endoprosthesis is completely smooth, and (3) there are no reports about microembolisation of PTFE endoprosthesis so far. The clamping period was monitored clinically by asking the patient to move the contralateral arm. Transcranial Doppler monitoring was not applied during the clamp period because of its low sensitivity, particularly in women. Limitations are that the underlying arteriopathy was not histologically confirmed and that the follow up period was short.

In conclusion, carotid stenting may present an alternative to endarterectomy in ICA stenosis due to fibromuscular dysplasia when ischaemic events persist despite full antithrombotic therapy. To date, the usefulness and the ratio risk/benefit of such a procedure in a group of asymptomatic patients is unclear and needs to be evaluated.

We are grateful to Dr P Samec for providing the preoperative angiographies.

1 Gomez CR. The role of carotid angioplasty and stenting. Semin Neurol 1998;18:501-11.

2 DeOcampo J, Brillman J, Levy DI Stenting: a new approach to carotid dissection. F Neuroimaging 1997;7:187-90.

3 Manninen HI, Koivisto T, Saari $\mathrm{T}$, et al. Dissecting aneurysms of all four cervicocranial arteries in fibromuscular dysplasia: treatment with self-expanding endovascular stents, coil embolization, and surgical ligation. Am 7 Neuroradiol 1997;18:1216-20.

4 Starr DS, Lawrie GM, Morris GC Jr. Fibromuscular disease of carotid arteries: long term results of graduated internal dilatation. Stroke 1981;12:196-9.
5 Kubis N, Von Langsdorff D, Petitjean C, et al. Thrombotic carotid megabulb: fibromuscular dysplasia, septae, and schemic stroke. Neurology 1999;52:883-6.

6 Schievink WI, Bjornsson J, Piepgras DG. Coexistence of fibromuscular dysplasia and cystic medial necrosis in a patient with Marfan's syndrome and bilateral carotid artery dissections. Stroke 1994;25:2492-6.

7 Moreau P, Albat B, Thevenet A. Fibromuscular dysplasia of the internal carotid artery: long-term surgical results. $\mathcal{f}$ Cardiovasc Surg 1993;34:465-72.

8 Tan AK, Venketasubramanian $\mathrm{N}$, Tan $\mathrm{CB}$, et al. Ischemic stroke from cerebral embolism in cephalic fibromuscular dysplasia. Ann Acad Med Singapore 1995;24:891-4.

9 Schievink WI, Meyer FB, Parisi JE, et al. Fibromuscular dysplasia of the internal carotid artery associated with alpha-antitrypsin deficiency. Neurosurgery 1998;43:229-34.

10 Sturzenegger $M$, Huber P. Cranial nerve palsies in spontaneous carotid artery dissection. $\mathcal{F}$ Neurol Neurosurg Psychiatry 1993;56:1191-9.

11 Lanzino G, Mericle RA, Lopes DK, et al. Percutaneous transluminal angioplasty and stent placement for recurrent carotid artery stenosis. $\mathcal{F}$ Neurosurg 1999;90:688-94.

12 Wholey MH, Wholey M, Bergeron P, et al. Current global status of carotid artery stent placement. Cathet Cardiovasc Diagn 1998;44:1-6.

13 Henry M, Amor M, Masson I, et al. Angioplasty and stenting of the extracranial carotid arteries. F Endovasc Surg 1998;5:293-304.

14 Mathur A, Roubin GS, Iyer SS, et al. Predictors of stroke complicating carotid artery stenting. Circulation 1998;97: 1239-45.

15 Teitelbaum GP, Lefkowitz MA, Giamotta SL. Carotid angioplasty and stenting in high-risk patients. Surg Neurol 1998;50:300-12.

16 Hobson RW 2nd, Goldstein JE, Jamil Z, et al. Carotid restenosis: operative and endovascular management. $\mathcal{F}$ Vasc Surg 1999;29:228-38.

17 Calvey TA, Gough MJ. A late complication of internal carotid artery stenting. $\mathcal{F}$ Vasc Surg 1998;27:753-5.

18 Naylor AR, Bolia A, Abbott RJ, et al. Randomized study of carotid angioplasty and stenting versus carotid endarterectomy; a stopped trial. F Vasc Surg 1998;28:32634.

19 Bettmann MA, Katzen BT, Whisnant J, et al. Carotid stenting and angioplasty: a statement of healthcare professionals from the Councils on Cardiovascular Radiology, Stroke, Cardiovascular Surgery, Epidemiology and Prevention, and Clinical Cardiology, American Heart Association. $\mathcal{f}$ Vasc Interv Radiol 1998;9:3-5. 